\title{
An Analysis of the Two-Dimensional Heat Transfer Model of the PCR Instrument Pedestal
}

\author{
Aiguo Zhou, Bei Liu, Xiufeng Xu and Wanli Li \\ Tongji University, Shanghai, China
}

\begin{abstract}
By analyzing the heat transfer situation of the polymerase chain reaction (PCR) instrument pedestal, a mathematical model to reflect the temperature field function of the pedestal is proposed, and then this model is simplified to obtain a simple two-dimensional heat transfer model. According to the results of the simplified mathematical model, the temperature field of the PCR instrument pedestal is calculated and simulated. And according to the calculation of two-dimensional temperature field, the influence of various parameters the on the pedestal's temperature field is analyzed when the instrument is working in an uneven heating mode.
\end{abstract}

Keywords-PCR instrument; two-dimensional temperature field; mathematical model; various parameters

\section{INTRODUCTION}

Polymerase Chain Reaction (Polymerase Chain Reaction instrument, PCR instrument) is the basis of the molecular biology of instruments, the experiment requires its pedestal temperature gradient can be more and the controlling of heating can be more precise. The heat source of the PCR instrument discussed in this article is the semiconductor refrigerator thermoelectric cooler, (TEC) located at the bottom of the pedestal. When their working modes are uniform, pedestal's temperature field is only related to time and the height from the cooler. For one dimensional temperature fields, there have been many studies on the model. When the coolers at the bottom of the pedestal in different working modes, due to the different transfer of heat, pedestal's temperature field not only related to time and height, but also the heat flux of the coolers. It means the temperature field is a two-dimensional temperature field. This paper mainly analyzes this model.

\section{The Basic Principle of Heat Transfer}

\section{A. Fourier's Law}

The Fourier's law was proposed by B.J.Fourier (Baron Jean Baptiste Joseph Fourier), according to a large number of experimental phenomena about the fundamental laws of heat flow and temperature field. For isotropic material it can be expressed as: heat flow is proportional to the temperature gradient, in the opposite direction. Here the temperature gradient is the direction of the temperature rising, but the heat should flow on the direction of the temperature decrease. Here is the mathematical form of the Fourier's law.

$$
\mathbf{q}=-\lambda \operatorname{grad} t
$$

$\lambda$ is the material coefficient of thermal conductivity, the unit is $\mathrm{W} \cdot(\mathrm{m} \cdot \mathrm{K})^{-1}, \mathrm{q}$ as the heat flow, $\mathrm{t}$ for the temperature field function.

\section{B. Heat Transfer Differential Equation}

For analyzing the temperature field distribution, according to the law of conservation of energy and Fourier's law, we can get the heat transfer differential equation of solid internal:

$$
\frac{\partial t}{\partial \tau}=a \nabla^{2} t+\frac{q_{v}}{\rho c}
$$

$\mathrm{t}$ as the temperature field function, is a function of time $\tau$ and space coordinates, $a=\lambda /(\rho \cdot c)$ is material thermal diffusivity, $\rho$ means density material, $\mathrm{c}$ means the volume of material heat capacity, $q_{v}$ means the heating rate of the inside heat source, said unit per unit time per unit volume of heat yield, $\nabla^{2}$ is the Laplace operator.

\section{Instruction of HeAt Transfer Model}

\section{A. Establishment of Heat Transfer Model}

Integrated physical model of the PCR instrument is shown in Figure 1.

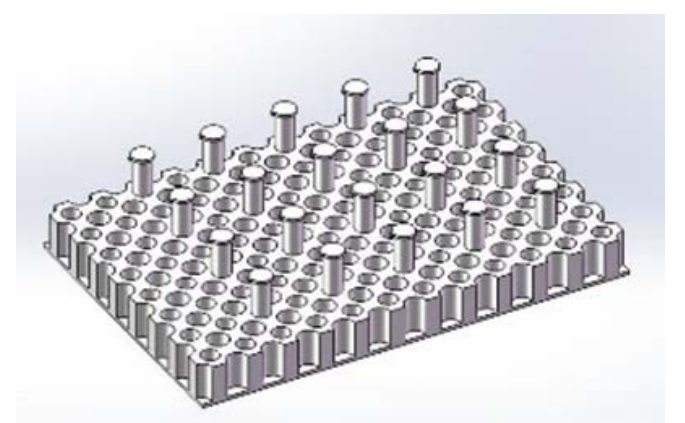

FIGURE I. THE PHYSICAL MODEL OF THE PCR INSTRUMENT

There are two thermoelectric coolers (TEC) located at the bottom of the pedestal, which are used as heat source, 
and the pedestal has no internal heat source. According to the actual heating conditions, while both of thermoelectric coolers are placed at the bottom of the base evenly. It can be considered that thermoelectric coolers cover the bottom of the base and contact well with the pedestal, ignoring the thermal contact resistance.

Meanwhile considering that both thermoelectric coolers heat flows the same on the width direction, Width dimension can be also ignored. Therefore, the base can be seen as a two-dimensional heat transfer model of planar sheet, as shown in figure 2 .

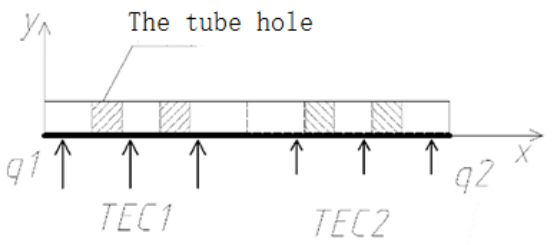

FIGURE II. THE THERMAL MODEL OF THE PEDESTAL

Among four borders of the planar sheet model, the bottom border contacts with the thermoelectric coolers in order to have heat exchange, so its boundary condition is determined by thermoelectric cooler working conditions, in consequence the boundary condition can be regarded as the second boundary condition, heat flux boundary.

The remaining three borders have heat exchange by air convection, heat flux is relatively small and can be further reduced by thermal insulation measures, so the boundary condition of these three borders can be simplified as the adiabatic condition.

The initial temperature of the base is uniform, and the differential equation of heat transfer is a linear equation, so the initial temperature can be regarded as the zero reference point, while the initial temperature of the base can be defining as 0 .

However, due to the existence of the tube holes and test tube reagents on the base, it is not uniform in the pedestal, which means, the thermal conductivity coefficient and the geometry of the base model is not uniform. In order to realize the uniformity of the thermal conductivity coefficient and the geometry of the base model, the holes and test tubes on the pedestal are considered to be the same material as the pedestal.

Due to the different thermal conductivity coefficient of test tube, tube holes and the pedestal material, actually there will be error in this process. Thus there is a virtual heat source $q_{v}$, which is used to eliminate the error. In this way the mathematical description of the model is

Differential equation: $\frac{\partial t}{\partial \tau}=a\left(\frac{\partial^{2} t}{\partial x^{2}}+\frac{\partial^{2} t}{\partial y^{2}}\right)+\frac{q_{v}}{\rho c}$

Boundary condition:

$$
\begin{gathered}
x=0,0 \leq y \leq d, \tau>0, \frac{\partial t}{\partial x}=0 \\
x=l, 0 \leq y \leq d, \tau>0, \frac{\partial t}{\partial x}=0 \\
y=0,0 \leq x \leq l, \tau>0,-\lambda \frac{\partial t}{\partial y}=q(x) \\
y=d, 0 \leq x \leq l, \tau>0, \frac{\partial t}{\partial y}=0
\end{gathered}
$$

Initial condition: $\tau=0, \mathrm{t}=0$

$q_{v}$ is used to compensate thermal difference, which is caused by different thermal conductivity coefficient between tube holes and the pedestal. It equals to the same external condition, which means, in the condition of real- pedestal temperature field, the absorbed thermal difference between base material and tube reagents.

\section{B. Model Simplification}

This paper mainly discusses the influence of each parameter on the temperature field of the base.

In the model above, there is functional relationship between the virtual heat source $q_{v}$ and the base temperature field. Suppose the temperature of the base is $t$, unit step temperature response of tube holes is $t_{1}$, and the base material temperature response under the condition of the same geometry is $t_{2}$, thus $q_{v}$ can be expressed as:

$$
q_{v}=t\left(\frac{\partial t_{2}}{\partial \tau}-\frac{\partial t_{1}}{\partial \tau}\right)
$$

Considering of the operation complexity in the virtual heat source, as well as the pedestal temperature field effects on the virtual heat source, and the value of the heat source is direct proportion to the pedestal temperature field, when discussing influence factors of the base temperature field, each factor influence through the virtual heat source is proportion to the temperature field itself, therefore the influence of the virtual heat source can be ignored for now.

When two pieces of thermoelectric coolers work with different efficiency, the heating mode can be expressed in Figure 3. 


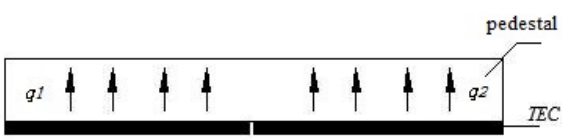

FIGURE III. THE HEATING MODE

Define the left side of the thermoelectric cooler working flow rate as $q_{1}$, the right side working flow rate as $q_{2}$.

According to the simplified two dimensional heat transfer model above, when the pedestal length is 1 and the height is $\mathrm{D}$, the temperature field function of the pedestal can be expressed as the function solution of the following model.

Differential equation: $\frac{\partial t}{\partial \tau}=a\left(\frac{\partial^{2} t}{\partial x^{2}}+\frac{\partial^{2} t}{\partial y^{2}}\right)$

Boundary condition:

$$
\begin{gathered}
x=0,0 \leq y \leq d, \tau>0, \frac{\partial t}{\partial x}=0 \\
x=l, 0 \leq y \leq d, \tau>0, \frac{\partial t}{\partial x}=0 \\
y=0,0 \leq x \leq l, \tau>0,-\lambda \frac{\partial t}{\partial y}=q(x) \\
y=d, 0 \leq x \leq l, \tau>0, \frac{\partial t}{\partial y}=0
\end{gathered}
$$

\section{THE SOlution OF THE MOdEL}

\section{A. Solution of the Green's Function}

The Green's function [1] can be used to solve this heat conduction model. The long time Continue heat flux can be seen as a superposition of multiple local within a single point of instantaneous heat source. Then the temperature field of the whole model is a superposition of temperature fields of all instantaneous point heat sources. The intensity of this point heat source can be chosen as $p^{c}$,containing the heat needed for higher unit volume object $1{ }^{\circ} \mathrm{C}$.Here we introduce the $\delta$ function. This function satisfies $\delta(x)=\left\{\begin{array}{c}0, x=0 \\ \infty, x \neq 0\end{array}\right.$ and $\int_{-\infty}^{\infty} \delta(x)=1$. Then the inner heat source can be expressed as $\rho c \delta\left(x-x^{\prime}\right) \delta\left(y-y^{\prime}\right) \delta\left(\tau-\tau^{\prime}\right)$. The Green's function, $G\left(x, y, \tau, x^{\prime}, y^{\prime}, \tau^{\prime}\right)$, is the temperature field function of a single instantaneous heat source. It says the temperature field produced by the instantaneous heat source at $x=x^{\prime}, y=y^{\prime}$ when $\tau=\tau^{\prime}$. Obviously, the Green's function to satisfy the following equation:

$$
\frac{\partial G}{\partial \tau}=a\left(\frac{\partial^{2} G}{\partial x^{2}}+\frac{\partial^{2} G}{\partial y^{2}}\right)
$$

$$
\begin{gathered}
\tau=\tau^{\prime}, G=\delta\left(x-x^{\prime}\right) \delta\left(y-y^{\prime}\right) \\
x=0 \text { or } x=l, 0 \leq y \leq d, \tau>0, \frac{\partial G}{\partial x}=0 \\
y=0 \text { or } y=d, 0 \leq x \leq l, \tau>0, \frac{\partial G}{\partial y}=0
\end{gathered}
$$

Using the method of separation of variables, we can obtain $G=G_{x} \cdot G_{y}$;

$$
G_{x}=\frac{1}{l}+\frac{2}{l} \cdot \sum_{n=1}^{\infty} e^{-a \beta_{n}^{2}\left(\tau-\tau^{\prime}\right)} \cos \beta_{n} x^{\prime} \cos \beta_{n} x, \quad \beta_{n}=\frac{n \pi}{l}
$$

$$
G_{y}=\frac{1}{d}+\frac{2}{d} \sum_{m=1}^{\infty} e^{-a \gamma_{m}^{2}\left(\tau-\tau^{\prime}\right)} \cos \gamma_{m} y^{\prime} \cos \gamma_{m} y, \quad \gamma_{m}=\frac{m \pi}{d}
$$

\section{B. Solution of the Model}

The heat flux on the bottom boundary of the model can be seen as a number of heat points on the time series. Considering the transformation between the boundary heat flux intensity and the heat intensity when obtaining the Green's function, the whole temperature field can be writing as

$$
t(x, y, \tau)=\frac{1}{\rho c} \int_{0}^{\tau} \int_{0}^{l} q_{x} G\left(x, y, \tau, x^{\prime}, y^{\prime}=0, \tau^{\prime}\right) d x^{\prime} d \tau^{\prime}
$$


Into $q_{x}$ and the Green's function $G\left(x, y, \tau, x^{\prime}, y^{\prime}, \tau^{\prime}\right)$ and we can get the solution.

$$
\begin{array}{r}
\mathrm{t}=t_{\tau}+t_{x}+t_{y}+t_{x y} \\
t_{x}=\frac{a\left(q_{1}+q_{2}\right)}{2 \lambda d} \tau
\end{array}
$$

It says the integral average temperature rise of the pedestal under the action of continued heat flow.

$$
t_{x}=\frac{2\left(q_{1}-q_{2}\right)}{\lambda d l} \sum_{n=1}^{\infty} \frac{1-e^{-a \beta_{n}^{2} \tau}}{\beta_{n}^{3}} \sin \frac{\beta_{n} l}{2} \cos \beta_{n} x
$$

It says the of the change of the temperature field in the $\mathrm{x}$ direction caused by the uneven heat flux;

$$
t_{y}=\frac{q_{1}+q_{2}}{\lambda d} \sum_{m=1}^{\infty} \frac{1-e^{-a \gamma_{m}^{2} \tau}}{\gamma_{m}^{2}} \cos \gamma_{m} y
$$

It says the change of the temperature field in the $y$ direction when the heat flows from the bottom to the top surface;

$$
t_{x y}=\frac{4\left(q_{1}-q_{2}\right)}{\lambda d l} \sum_{n=1}^{\infty} \sum_{m=1}^{\infty} \frac{1-e^{-a\left(\beta_{n}^{2}+\gamma_{m}^{2}\right) \tau}}{\beta_{n}\left(\beta_{n}^{2}+\gamma_{m}^{2}\right)} \sin \frac{\beta_{n} l}{2} \cos \beta_{n} x \cos \gamma_{m} y
$$

It says the change of temperature field caused by the uneven heat which flows from the bottom to the top surface.

\section{The Simulation Analysis}

The material of the pedestal is aluminum. We can check its coefficient of thermal conductivity $\lambda=238 W(m \cdot K)^{-4}$, the thermal diffusivity $\lambda=238 \mathrm{~W} \cdot(\mathrm{m} \cdot \mathrm{K})^{-1}$. The length of the base is $l=0.12 \mathrm{~m}$, and height is $d=0.01 \mathrm{~m}$. Take $q_{1}=1 \times 10^{5} \mathrm{~W} \cdot \mathrm{m}^{-2}$ and $q_{2}=0$, namely the cooler on the left side works and the cooler on the right side doesn't work. Then we can get the temperature field of the pedestal on this working mode. To discuss the effect of the influence factors on the temperature field of the pedestal, we can adopt the way of controlling variables by drawing the response curve of the temperature field about each single factor. The result is shown in figure 4 :

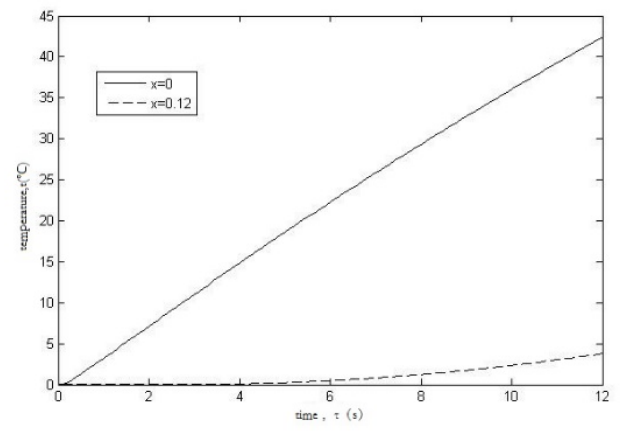

FIGURE IV(A). THE RESPONSE OF THE TEMPERATURE FIELD OVER TIME ON THE TOP SURFACE

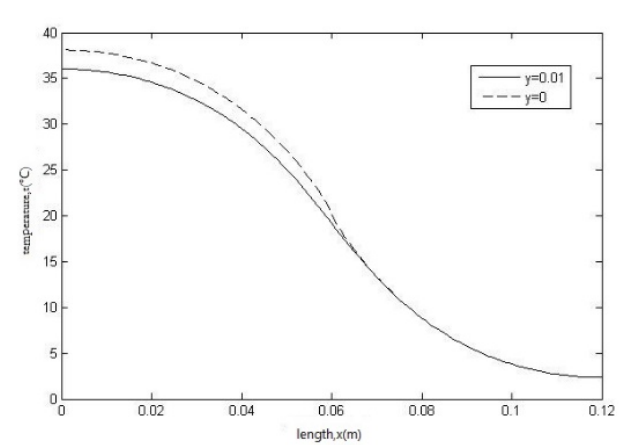

FIGURE IV(B). THE DISTRIBUTION Of TEMPERATURE FIELD ON X DIRECTION WHEN $\tau=10$ s

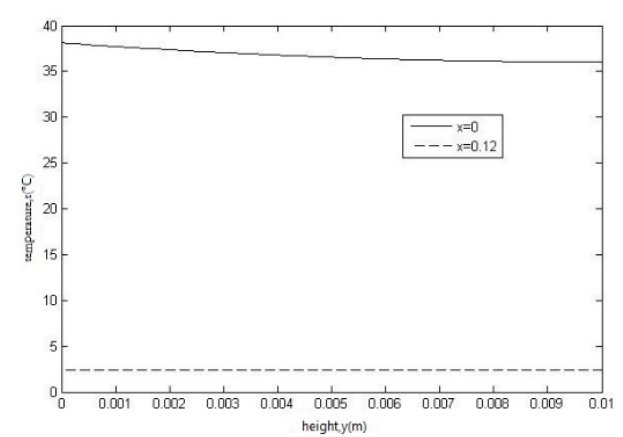

FIGURE IV(C). THE DISTRIBUTION Of TEMPERATURE FIELD ON Y DIRECTION WHEN $\tau=10$ s

The response of the pedestal's temperature field over time can be seen from the figure $4 \mathrm{a}$. On the left side, the TEC works and provides heat for the pedestal. The pedestal's temperature increases and is roughly proportional to time after a short delay of thermal conductivity. The right side of the TEC doesn't work. Relying mainly on the internal heat transfer heat, the pedestal's temperature on the right side is significantly lower than the left and increases slower with longer time delay.

The figure $4 \mathrm{~b}$ says the pedestal's temperature field distribution along the length of $\mathrm{x}$ direction. Due to the constant supply of heat on the left side, the temperature on the left significantly higher than that on the right side where there is no heat source. In the central, due to the effect of the heat flow in the pedestal itself, which cooling the hot left side and heating the cold right side, temperature difference decreases.

The figure $4 \mathrm{c}$ says the pedestal's temperature field distribution along the height direction $y$. Because of the heat source located at the bottom of the pedestal on the left side, the temperature of the left side has a certain temperature drop gradient along the height direction. The right side of the pedestal is heated by the heat flux flows from the left side. So it's less affected by the height.

\section{SUMMARY}

Based on PCR instrument pedestal heating mode and the working situation, using virtual heat source instead of the 
original base in geometric shapes and coefficient of thermal conductivity inhomogeneity, this article established a mathematical model for the temperature field of the PCR instrument pedestal. And according to the simplified model, the pedestal's temperature field is calculated. The pedestal's temperature field's distribution and its response over time is also discussed. The pedestal's temperature generally is proportional to the heat flux and heating time, but the distribution of temperature field is also effected by the TEC's working conditions and the heat flowing in the pedestal. On the one hand, the pedestal's temperature is proportional to the heat flux the TEC supplied. There is high temperature in the TEC heating part and low temperature in the no heating part. And the temperature is higher in where is nearer to the heat source. Therefore, the temperature difference is formed within the pedestal. On the other hand, the effect of heat flowing will try the best to reduce the difference in temperature between the adjacent parts. The bigger the temperature difference, the more obvious the effect is, which makes the base temperature field to keep smooth. This analytical simulation of temperature field model for PCR instrument pedestal may provide some reference data to temperature controlling.

\section{REFERENCES}

[1] Jia Li, Zhao Honggang, Qian Xinghua. Heat Transfer Theory [M]. Beijing: The High education Publishing House, 2008.

[2] Yang Qiangsheng, Pu Baorong. Heat Transfer Theory [M]. Shanghai: Shanghai Jiaotong University Publishing House, 1996.

[3] Harold Levine. Partial. Differential Equations [M]. Beijing: higher education Publishing House, 2007.

[4] Mao He, Chen Zhangwei, Huang Jing, etc. PCR temperature field model of research and validation [J]. Journal of Zhejiang university, 2013, 47 (9); 1619-1624.

[5] Yang Xiangxiang Weng Rongzhou. Two-dimensional finite element analysis of the fin heat transfer [J]. Journal of Huaqiao university, 1988, 9 (4); 509-513.

[6] Huang Jing, Chen Zhang, Yao Yinghao, etc. A quantitative PCR thermal cycling system temperature uniformity of the finite element simulation [J]. Journal of Instruments and Meters, 2010, 31 (5); 11421147. 